OPEN ACCESS

Edited by:

Michitaka Hirose,

The University of Tokyo, Japan

Reviewed by:

Phil Lopes,

Universidade Lusófona, Portugal

Gershon Dublon,

Massachusetts Institute of

Technology, United States

*Correspondence:

Dion Willis

dion.willis@port.ac.uk

Specialty section:

This article was submitted to Virtual Reality and Human Behaviour, a section of the journal

Frontiers in Virtual Reality

Received: 15 December 2020

Accepted: 12 May 2021

Published: 28 May 2021

Citation:

Willis $D$, Stevens $B$ and Powell $W$ (2021) Visual Capture of a Tactile Sensation is Influenced by Repeated, Structured Exposure of a Visual

Stimulus in Virtual Reality.

Front. Virtual Real. 2:642061.

doi: 10.3389/frvir.2021.642061

\section{Visual Capture of a Tactile Sensation is Influenced by Repeated, Structured Exposure of a Visual Stimulus in Virtual Reality}

\author{
Dion Willis ${ }^{1 *}$, Brett Stevens ${ }^{1}$ and Wendy Powell ${ }^{2}$ \\ ${ }^{1}$ Creative and Cultural Industries, Creative Technologies, University of Portsmouth, Portsmouth, United Kingdom, ${ }^{2}$ MindLabs, \\ Department of Cognitive Science and Artificial Intelligence, Tilburg School of Humanities and Digital Sciences, Tilburg University, \\ Tilburg, Netherlands
}

Phantom limb pain is commonly known as a neurological condition, where an amputee will continue to feel a limb that is no longer present in a painful fashion. Virtual mirror therapy (VMT) has been suggested as a method for alleviating phantom limb pain. The inclusion of tactile sensation in VMT has shown to be beneficial; however, delivering a tactile sensation to a phantom limb, without the use of invasive procedures, can be difficult. The current approach for transferring a tactile sensation to a phantom limb is called visual capture. The ability to establish visual capture has been demonstrated in VMT applications. However, there is little research into whether an established visual capture effect can be relocated to a more distal location for phantom limb pain management. This paper investigates whether a passive vibrotactile sensation can be moved to a distal location from its veridical location using a series of distally located lights presented in either a random or a structured fashion. Eight non-amputee participants were tasked with localising a static tactile sensation on a virtual arm. These vibrotactile sensations were presented simultaneously with a visual light stimulus, either co-located or located distally at three different locations. Findings show that a tactile sensation without a visual stimulus was difficult for participants to localise; however, when a visual stimulus was added, they were better able to locate the veridical tactile position. The structured group exhibited a larger range of tactile relocation responses than the random group. However, this result was unreliable, with the majority of the responses situated at the vibrotactile actuator. There was a significant difference between the random and structured group's ability to retain a visual capture at the veridical vibrotactile location when the lights were located distally. The random group did not express a visual capture response when the lights were presented distally while the structured group did, suggesting the structured group developed a more robust association between the visual stimulus and the vibrotactile stimulus. Findings may be of use where increasing tactile acuity without significant alteration of a veridical location is a desired therapeutic outcome.

Keywords: Visuo-tactile, Multisensory, Virtual reality, Phantom limb pain, Rehabilitation, Visual capture, Tactile localisation, Tactile acuity 


\section{INTRODUCTION}

\subsection{Phantom Limb Pain, Visual Capture-Based Rehabilitation and Neural Plasticity}

Phantom limb pain (PLP) is a specific type of deafferentation pain which affects amputees. It is a neurological condition where a person will continue to feel a painful limb even if it is no longer there. The phantom limb will generally experience cramping, itching, freezing, or burning temperatures (Weeks et al., 2010). Similar symptoms have been expressed without loss of limb such as brachial plexus avulsion or stroke in which injury or damage is sustained in the brain (somatosensory cortex and motor) or peripheral nerves (Shankar et al., 2015). Deafferentation and phantom limb pain have been shown to be very complex, and effective treatment remains undecided due to low-level evidence in findings (Dunn et al., 2017). Cognitive treatments such as mirror box therapy have shown to be promising and small-scale studies have shown to have good efficacy for managing pain without the possible side effects that invasive or pharmacological treatments have presented (Richardson and Kulkarni, 2017).

Traditional mirror box therapy visually superimposes a reflection of their intact limb onto their phantom limb, using mirrors. The mechanism utilised by mirror box therapy is a psychological principle called visual capture, sensory calibration, or the ventriloquism effect, and takes advantage of human's natural tendency to rely on visual cues over other modalities (Carey et al., 2019). If a non-visual stimulus is presented simultaneously and in a congruent manner with a visual stimulus, the visual stimulus will generally capture properties of the other modality, such as positional information. This visual illusion allows a clinician to remedy the pain in their phantom limb by manipulating or stimulating their intact limb, even though the phantom limb is not directly accessible (Carey et al., 2019). The current understanding for the emergence of phantom limb pain and the approach to treatment, is the brains natural plasticity and reorganisation after injury and amputation Flor and Diers (2009). Thus, the goal of mirror therapy is to stimulate affected areas of the brain such as the motor cortex and the somatosensory cortex via the intact limb or representation of the affected limb. Stimulation in the motor and somatosensory cortex is stated to reverse or alleviate the structural neural reorganisation that takes place in amputees or patients suffering from deafferentation pain (Flor and Diers, 2009; Kuner and Flor, 2017).

Cognitive treatments have been augmented from analogue means using mirrors to a more technological means using virtual reality or augmented reality. Virtual mirror therapy (VMT) aims to recreate the lost or deafferented limb in a virtual environment using head mounted displays or desktop monitors. This is accomplished by either reflecting the intact limb using hand trackers such as motion capture equipment or extending virtually recreating the limb using myoelectric sensors, which take the small electrical efferent signals in the in residual limb or adjacent muscles to drive the movements of the virtual limb (OrtizCatalan et al., 2014; Wake et al., 2015; Sano et al., 2016). This translation to more technological means allows more tailored experiences, which have resolved some of the issues faced in traditional forms, such as the ability to customise the appearance of the limb for better embodiment, or to extend the virtual limb using the residual limb in the case of bilateral amputees (Dunn et al., 2017; Perry et al., 2018). Akin to traditional methods, the effectiveness of virtual mirror therapy has shown to increase with the inclusion of additional modalities (Wake et al., 2015; Sano et al., 2016; Osumi et al., 2020).

\subsection{Virtual Mirror Therapy Utilising Visuo-Tactile Methods}

Application of virtual mirror therapy has focused on visualisation of the limb and creating proprioceptive exercises to alleviate cramping and postural issues, however, these sensations constitute only a portion of the painful experience's amputees suffer from (Pirowska et al., 2014). Burning, freezing, shooting pain and other paraesthesia are prevalent experiences associated with deafferentation pain and phantom limb pain and findings suggest that these painful phenomena are not as well managed by proprioceptive based exercises such as virtual mirror therapy (Osumi et al., 2019). This may be due to paraesthesia generally relating to the somatosensory cortex rather than the motor cortex. Although there is an overlap and cooperation with these cortex's, activation within the motor cortex has shown to be better triggered when performing a motor task, such as the exercises seen in virtual mirror therapy applications (Zhang et al., 2018). In contrast, the somatosensory cortex activates much more with tactile sensory stimuli, such as vibrations from texture and temperature differences (Purves, 2018). Research exploring the introduction of tactile sensation in a virtual mirror therapy protocol have shown promising results and Sano et al. (2016) has incorporated tactile sensation alongside audio cues into a common VMT protocol via the inclusion of vibrotactile actuators at the fingertips of the patient's intact fingers. Their application involves participants performing an active grasping task with their intact limb, which is reflected to where the phantom limb is experienced. When the participants grasp a virtual object in the application, tactile sensations are provided to the fingertips, alongside an audio cue, giving the illusion that the phantom limb is now touching the virtual objects. Sano et al. (2016) found that immediate pain was decreased to a greater degree when compared to the visual representation of the limb alone. This type of tactile stimulation realigns VMT with traditional mirror therapy in which the limb is visualised as well as manipulated in a proprioceptive and tactile fashion (Finn et al., 2017).

Although Sano et al. (2016)'s method has shown to be useful, there are many areas still to be explored. There is reason to believe a passive tactile sensation where a person experiences a touch that was not intentional or may not be expected, may invoke a greater or at least a different neural response in the somatosensory cortex, thus invoking alternative therapeutic properties (Ackerley et al., 2012; Simões-Franklin et al., 2011). In addition, Sano et al. (2016)'s protocol alongside tradition mirror therapy's use of contralateral mirroring of tactile sensation may not be feasible 
or appropriate for specific demographics such as bilateral amputees in which vibrotactile actuators cannot be utilised due to an intact limb not being present. Although there are other ways of virtually recreating the phantom limb's proprioception and visual characteristics using technology such as myoelectric sensors, that do not require an intact limb (Ortiz-Catalan et al., 2014). There is little research currently investigating how to deliver a tactile sensation to a portion of the limb without directly mirroring the sensation from one side of the body to the other as mirror therapy and virtual mirror therapy demonstrates. With this in consideration, it could be suggested here that a method of ipsilateral, distal relocation of passive tactile sensation could show to be beneficial for use in specific amputee demographics, which this paper aims to provide some insight.

\subsection{Methods for Moving a Passive Tactile Sensation to a Distal Location}

Although projecting a tactile sensation distally on a limb using visual capture techniques may seem straightforward given the observations found in mirror therapy and virtual mirror therapy, there is evidence that suggests the contrary. Previous experimentation has shown, simply presenting a light in a synchronous yet spatially distal location to a vibrotactile sensation, can disrupt tactile localisation via a visual capture response, but has failed to demonstrate a gross relocation of tactile sensations (Willis et al., 2019). Related studies using vibration arrays and visual light stimulus in augmented reality have shown similar results being able to relocate tactile perceptions around $40 \mathrm{~mm}$. These results are interesting, but for phantom limb pain treatment, a more extensive relocation may be necessary (Niijima and Ogawa, 2014; Samad and Shams, 2018). In addition, there is also evidence that stimulation of the fingers, hand and forearm provided to one hand can also produce a neural response in section of the brain in responsible for processing touch of the opposite hand (Lamp et al., 2019). This suggests the visual capture response found in mirror therapy and virtual mirror therapy may be amplifying the results already present in normal perception allowing for an easier relocation of tactile sensation. These neural correlates have not yet been evidenced when referring touch to a different part of the limb. This may mean that distally relocating the tactile sensation may have some limiting capabilities compared to mirroring contralaterally.

Visual capture responses have been found to have long lasting effects that may provide insight for overcoming some of the limitations found in ipsilateral, distal, tactile relocation. These lasting effects are referred to as the ventriloquism aftereffect. The ventriloquism aftereffect details how a visual capture response can remain even though the visual stimulus that initially elicited it is no longer present Samad and Shams (2018). Unlike a visual capture response, the ventriloquist aftereffect has shown to be more flexible and mutable (Bosen et al., 2017). Bosen et al. (2017) discusses the how visual capture and the ventriloquism after effect may have separate neural mechanisms meaning they may not hold some of the neural limitations. Bosen et al. (2017) has demonstrated the ventriloquism aftereffect can be augmented and can accumulate after repeated exposure to a visuotactile pairing. Using a accumulated structured exposure to a visuotactile pairing to drive multiple ventriloquist aftereffects may be harnessed for a method of ipsilateral, distal relocation of passive tactile sensation for use in phantom limb pain treatment.

\section{MATERIALS AND METHODS}

The purpose of this study is to investigate whether a structured repeated exposure to a visual stimulus can relocate a passive tactile stimulus distally from the forearm to the fingers.

\subsection{Hypothesis}

1. Visual capture of a passive vibrotactile sensation can be distally relocated to larger degree with structured presentation of distally located visual stimulus rather than randomly presented visual stimulus.

\subsection{Participants}

A total of eight non-amputee participants were used in this study (five females, three males). Seven participants were right hand dominant, and 1 was Left hand dominant. Participants were recruited from the University of Portsmouth and were a mixture of staff and students. Age of the participants ranged from 19-40 years old. The exclusion criteria for this study were: Visual impairments which could not be corrected with visual aids such as glasses or contact lenses, visual field epilepsy, heightened tactile defensiveness, any known tactile discrimination deficits or recurrent/chronic pins and needles or numbness in the arms. None of the participants stated they felt any acute pain or were suffering from chronic pain. This study was reviewed by the University of Portsmouth ethics committee and given a favourable opinion following the University of Portsmouth guidelines. All participants gave written consent to take part in the study and for results to be published.

\subsection{Variables}

- Independent variable

- Visual stimulus presentation order (structured or random)

- Dependant Variable

- Section number in which the participants localised the tactile sensation

\subsection{Groups}

A between-groups study design was implemented. Participants were randomly allocated to one of two groups; a perceptually random group or a structured presentation group. These groups corresponded to the order that the participants viewed the visual light stimulus. All participants, no matter the group, conducted an initial tactile localisation without the presence of a visual light stimulus. This provided an initial baseline measurement for where participants localised the vibration actuators on there arm in regard to the virtual arm.

Participants in both the perceptually random group and the structured group experienced a vibrotactile stimulus in a consistent location on the arm and a temporally synchronous 


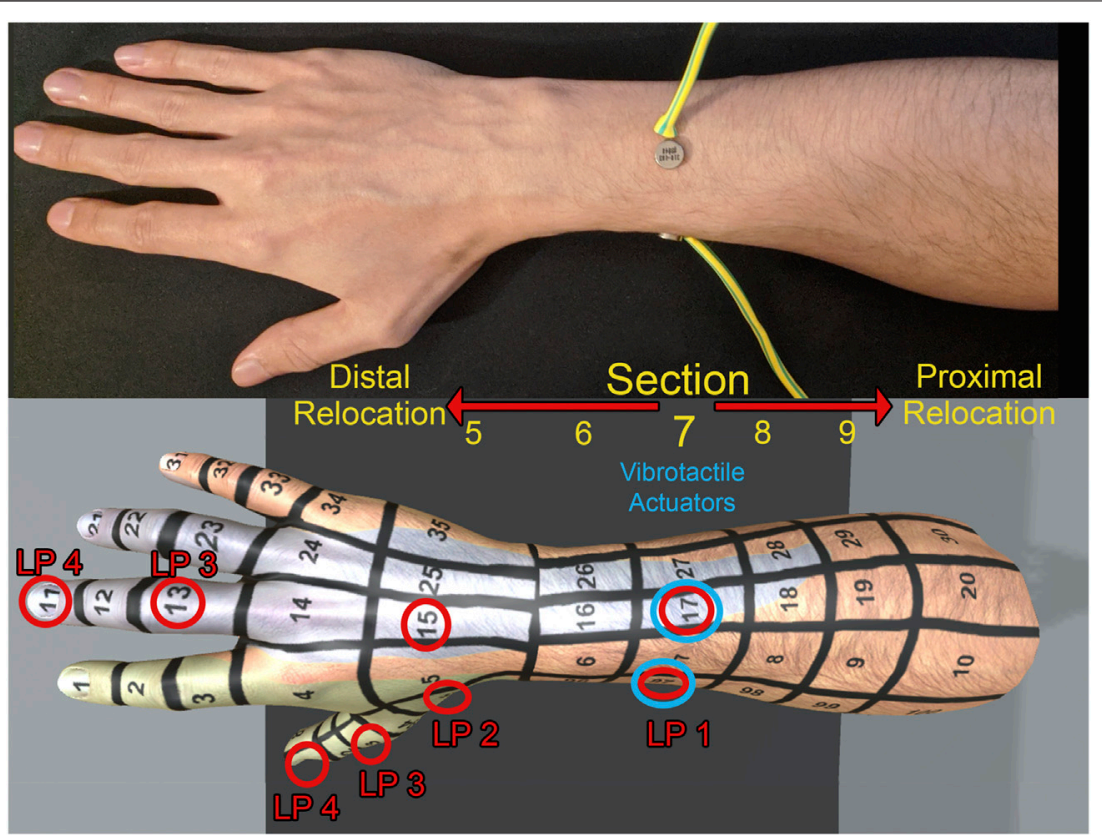

FIGURE 1 | Physical set-up of the vibration actuators on the arm (A) with the virtual fair skin male arm identifying light positions (B). The physical vibration actuators were placed at section 17 and $97 . \mathrm{LP}=$ Light position. Positions of the lights are labeled in red and position of the physical vibration actuator labeled in blue. The arm models had a blue and green color map applied which signaled dermatomes and were used for verification of verbal response.

light stimulus. The visual light stimulus was presented either colocated or distally separated in three locations down the arm. The perceptually random group was presented the light in one of the four possible locations randomly each exposure. In contrast, the structured group experienced the lights, and vibrations initially co-located on the forearm and becoming more distally decoupled in discrete jumps in one location.

This study initially included an extra variable which was investigating whether dermatomes influenced the results of the visuo-tactile perception. It was hypothesised that there would be differences between whether the vibration motor was placed on a site that had multiple overlapping dermatomes or a single dermatome. This warranted the placement of two vibration actuators instead of just one. Participants therefore experienced the same conditions depending on the group they were allocated on both the overlapping dermatome and the single dermatome (Lee et al., 2008). There was a break of 5 min between experiencing the overlapping dermatome vibration sequence and the single dermatome sequence to avoid any carry over effect. When data was examined this variable was found to be innocuous and displayed no significant differences between the sites. Data was compiled in the analysis and reassessed to increase power. This meant the amount of exposures in each location was doubled from 8 to 16 exposures. See findings section.

\subsection{Physical Set Up}

Participants had two vibration actuators attached to the dorsal side of their forearms (Figure 1). These vibration actuators were separated on the lateral/medial plane of the arm but were proximally/distally in line. Medical adhesive was used to attach the vibration actuators. Each vibrotactile sensation experienced by the participants lasted for $1 \mathrm{~s}(230 \mathrm{~Hz}$ and $1.2 \mathrm{~g}$ amplitude). Placement of the vibration actuators was determined by measuring the participants' arms and adjusting the scale of the virtual arms to match. Measurements from the participants fingertips to their wrist was taken and a measurement from their wrist to their forearm created a scale factor for which the virtual model could be matched. Placement of the vibration actuators on the forearm was determined using the wrist in flexion as a reference point and measurements taken from the scaled virtual arm.

Due to the risk of participants remembering the positions of the vibration motors when they were applied to the skin, the lead researcher gave false indications that there may have been more motors attached to the arm. These false indications took the form of pressing the skin in random places along the forearm and hand. Placing inactive motors on the skin was proposed, however, results in Willis et al. (2019) showed that 7 of 16 people said people could faintly feel the presence of the vibration motors on the skin even when they were not vibrating. As participants were aware of the vibration actuators, it was suspected there was a chance to observe an unintended funnelling effect (Barghout et al., 2009). The funnelling effect can alter tactile localisation when two (or more) different locations simultaneously with different amplitudes can elicit phantom sensations in the space between (Lee et al., 2015). Instead, false presses on the skin were utilised to mask the true location as the tactile sensation from the presses should fade before the experiments measurements.

The vibration actuators were connected via wires to a core electronics platform worn on the participants back. Efforts were 
made to keep the wires away from the participants' arms by reinforcing the wires to give sufficient rigidity to trail away from the arms, ensuring that the only contact point on the participants were the attached vibration actuators. The vibrations were delivered through an Arduino mini pro, which communicated with unity via a serial cable. Each time the space bar was pressed by the lead researcher, a signal was sent to the Arduino issuing a command to a vibration motor to turn on and off.

\subsection{Virtual Environment}

The application was created in Unity and assets created in $3 \mathrm{ds}$ Max 2015. An Oculus Rift CV1 was used to display the virtual environment for the participant. A LEAP motion device was used to track the participants' hands and mapped the movement to the arms in the virtual environment. Tracking of the arms was checked before, and during for any overt latency issues which may have inhibited embodiment. None were present and participants did not report any when asked after the experiment. Participants were able to choose the appearance of the limb (male or female) and they had a choice of three different skin colors (fair, tan, and dark). Attached to these arms were eight virtual lights positioned in four locations on the arm (Figure 1). As the dermatome variable was removed due to having no significant effects on the results; and an overall focus on the distal/proximal localisation of the tactile sensation, the labeling denoting the section of where the lights were located have been standardised to use only the units. The units of the section corresponds to the distal/proximal location on the arm.

1. Located in section 7 co-located with the vibrotactile actuator on the forearm

2. Located in section $\mathbf{5}$ two sections distal from the vibrotactile actuator on the hand

3. Located in section $\mathbf{3}$ four sections distal from the vibrotactile actuator on the finger

4. Located in section $\mathbf{1}$ six sections distal from the vibrotactile actuator on the fingertip

The lights were switched off by default and when active remained present for $1 \mathrm{~s}$. The light was rendered from Unity's halo function with an overall luminosity spanning $1.5 \mathrm{~cm}$ in a spherical manner (decaying to zero from $1 \mathrm{~cm}$ ). Participants had a choice of either male or female arms. Sections were arranged in a grid format and followed anatomical landmarks such as fingers, wrist and knuckles. The arms were divided into 100 sections in total. These sections were not equal in size; however, each section was big enough for the vibration actuators and lights to fit into and were large enough to accommodate receptive fields (Purves, 2018). The sections ranged from 1 to 100 and wrapped around the arm. Numbers we ordered in a line from 1 (distal) to 10 (proximal) continued laterally around the arm. The number located in the sections of the grid were used by the participant to localise where they felt the vibration. A colored overlay was placed on the hands and forearms that represented the $c 6$ and $c 8$ dermatomes (this justification was not
TABLE 1 | Mann-Whitney $U$ test results comparing the tactile localisation between the random group and the structured group at the different light positions.

\begin{tabular}{lccc}
\hline & $\boldsymbol{U}$ value & $\boldsymbol{p}$ value & $\begin{array}{c}\text { Effect } \\
\text { size (R value) }\end{array}$ \\
\hline No light condition & 2021.500 & 0.896 & 0.01 \\
Light position 1 & 1680.500 & $0.019^{*}$ & -0.21 \\
Light position 2 & 1439.000 & $0.002^{*}$ & -0.274 \\
Light position 3 & 1551.500 & $0.014^{*}$ & -0.217 \\
Light position 4 & 1802.500 & 0.221 & -0.108
\end{tabular}

disclosed to participants). This colored overlay was for validation to mitigate human error in reporting, that was present during a pilot study of the study. Vocal verification of section with a color associated was encouraged. There is little evidence to suggest this coloring would influence relocation of the vibrotactile sensation, and upon inspection, there did not seem to be any influence.

\subsection{Task}

Conditions were triggered by the lead researcher. Once triggered, a combination of vibrations and/or lights were presented to the participant depending on the phase the participant was in. Each participant was exposed to the no light condition to provide a baseline tactile localisation. Participants then proceeded on their group's intervention, either perceptually random lights or structured lights. After each condition, participants were asked to verbally state the section number they felt the vibration. This verbal response comprised the dependant variable. They were also asked to give any other comments about the experience after each condition. The timing between each condition was up to the participants' speed in verbally stating the section and conveying experiential data. Due to the time delay between exposure and response, it is unlikely any apparent motion illusions were experienced (Ueda et al., 2008). Participants were asked to keep the entire virtual arm in view for the duration of the study. Participants had agency of moving their arms throughout the study but were encouraged not to make any sudden or large arm movements or rotations, this limited the amount the cables moved and kept the tracking stable. The lead researcher made visual observations to make sure this was the case.

Arm fatigue was alleviated by enforcing breaks after trials 20 , 40 , and 60 . This break was issued in the middle of a sequence not to disrupt the flow of the experiment and to limit any decay experienced in the possible ventriloquism aftereffect. Discomfort and pain have previously been shown to disrupt tactile acuity (Moseley et al., 2008). In order to mitigate this risk, participants were asked to lower their arms to their sides for a minimum of $30 \mathrm{~s}$ during the breaks, in order to recover from any arm or neck fatigue.

\section{ANALYSIS AND FINDINGS}

The dependant variable was the section number on the virtual arm that participants localised the vibration in. As the 


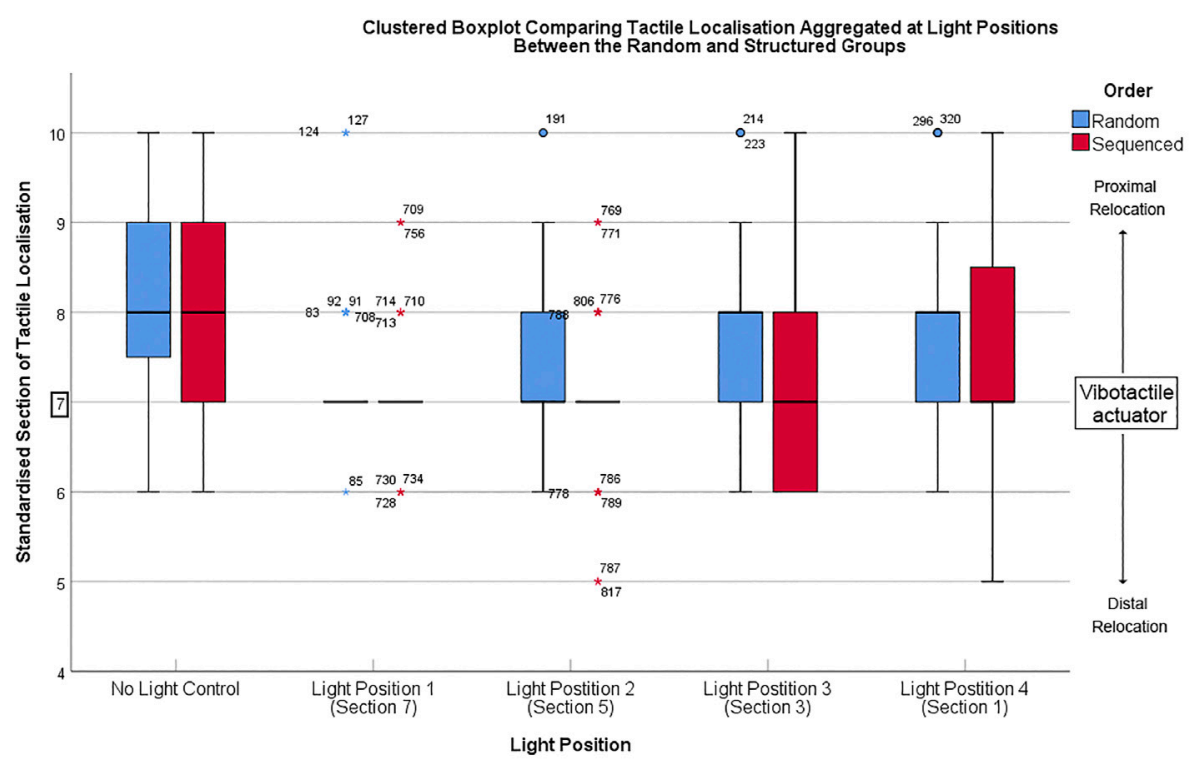

Y-Axis indicates the standardised number of the section participants localised the tactile sensation. The scale ranges from from 1 at the finger tips to 10 on the forearm.

FIGURE 2 |Y-Axis shows the standardised number of the section participants localised the tactile sensation. The scale ranges from 1 at the finger tips to 10 on the forearm.

hypothesis is focused on distal relocation of the tactile localisation, responses have been standardised to only contain information regarding proximal and distal movement. This means lateral/medial relocation information has been removed for this analysis. For example, a response of section 17 will be interpreted as section 7 , as the units of the section correspond to distal movement down the arm ( $1=$ Distal, $10=$ Proximal). This standardisation alongside the grid sections on the arm being varying sizes, meant all data collected could be treated ordinal in nature, thus making non-parametric tests most appropriate.

The study initially contained an extra variable investigating differences in tactile localisation between a vibration actuator placed in a position with overlapping dermatomes or a single dermatome, at the different light positions (see the section 2.4). Data was split isolating the Random group and the structured group so there was no influence of the presentation of lights. Tactile localisation during the no light condition and light positions was compared between the overlapping dermatome and single dermatome. A Levene's test showed data to be homogeneous across the light positions. A Mann-Whitney-U test was conducted and showed no significant difference of tactile localisation between the vibration sites at different light positions. No light (random), $p=0.765$, No light (structured), $p=0.429$, Light position 1 (Random), $p=0.08$, Light position 2 (random), $p=$ 0.14 , Light position 3 (random), $p=0.053$, Light position 4 (random), $p=0.081$, Light position 1 (structured), $p=0.44$, Light position 2 (structured), p250 $=0.05$, Light position 3 (structured), $p=0.746$, Light position 4 (structured), $p=0.672$.
TABLE 2 | Pairwise comparisons investigating differences in tactile localisation between the light positions within the random and sequenced group. Each row tests the null hypothesis that Sample 1 and Sample 2 distributions are the same. Asymptotic significance's (2-sided tests) are displayed. The significance level is 0.05. Significance values have been adjusted by the Bonferroni correction for multiple tests. The table should be read vertically for individual groups.

\begin{tabular}{lcccc} 
& \multicolumn{2}{c}{ Random } & \multicolumn{2}{c}{ Sequenced } \\
\hline & Test Stat & Adj. Sig & Test Stat & Adj. Sig \\
\hline No light control - Light position 1 & 34.0 & $0.000^{*}$ & 36.0 & $0.000^{*}$ \\
No light control - Light position 2 & 4.57 & 0.325 & 21.5 & $0.000^{*}$ \\
No light control - Light position 3 & 4.57 & 0.325 & 15.2 & $0.001^{*}$ \\
No light control - Light position 4 & 5.68 & 0.172 & 11.3 & $0.008^{*}$ \\
Light position 1 - Light position 2 & 8.69 & $0.032^{*}$ & 2.6 & 1.00 \\
Light position 1 - Light position 3 & 10.8 & $0.010^{*}$ & 5.68 & 0.172 \\
Light position 1 - Light position 4 & 10.8 & $0.010^{*}$ & 8.53 & $0.035^{*}$ \\
Light position 2 - Light position 3 & 0.13 & 1.00 & 0.64 & 1.00 \\
Light position 2 - Light position 4 & 0.13 & 1.00 & 1.86 & 1.00 \\
Light position 3 - Light position 4 & 0.07 & 1.00 & 0.32 & 1.00
\end{tabular}

\subsection{Differences in Tactile Localisation Between the Perceptually Random Group and Structured Group}

Tactile localisation was compared between the random and structured groups at the light positions (no light, Light position 1, Light position 2, Light position 3, Light position 4) using a Mann-Whitney $U$ tests (Table 1). 64 responses were recorded per group per location. When the light was not present on the arm, there were no significant differences between the 
random and structured groups $(p<0.05)$. There were significant differences in tactile localisation between the random and structured group when the light was present and located at position 1,2 , and $3(p<0.02)$. No significant differences were found when the light was located at the fingertips at position 4 $(p>0.05)$. Descriptive statistics were collected indicating the standardised section of tactile localisation at during the different conditions (Figure 2).

\subsection{Differences in Tactile Localisation Between the Light Positions Within Groups}

Pairwise comparison of tactile localisation between no light and the various light positions was compared within both the random and structured group using median tests (Table 2). The random group exhibited a difference in tactile localisation when the light was present at position 1, compared to the baseline localisation when the light was not present $(p=0.000)$. When the light was present at positions 2,3 , and 4 there were no significant differences compared to the baseline where no light was present $(p>0.05)$.

The structured group showed significant difference in tactile localisation when the light was located in all four positions (positions 1, 2, 3, and 4) compared to baseline condition where no light was present $(p<0.008)$. When the light was present in position 1 there was a significant difference in tactile localisation compared to light position $4(p=0.035)$. Tactile localisation showed no significant differences when the light was located at position 1 compared to light positions 2 and 3 $(p>0.05)$.

\section{DISCUSSION}

This study hypothesised that a structured presentation of lights positioned at increasingly distal locations of an arm would relocate a tactile sensation to a further degree than a random presentation of lights. Although significant differences have been found between the groups the results from this study do not support the stated hypothesis. Neither the random nor the structured group experienced a large displacement of the vibrotactile sensation as seen in virtual mirror therapy protocols. The groups demonstrated similar results regarding the overall distal displacement of the tactile perception on the arm. Both the random and structured groups initial perception of the vibrotactile stimulus; without an accompanying light stimulus, was proximally misplaced from the veridical vibration location (section 7 to section 8). When a light accompanied the vibrotactile stimulation co-located at section 7 participants calibrated their initially misplaced perception to where they now saw the light, signifying that a visual capture effect was observed. When the lights were positioned at more distal positions on the arm compared to the veridical vibrotactile site, the associative connection between the light and vibration creating the initial visual capture was not retained in a normal capacity. There were unexpected differences between how the random and structured group retained a visual capture situated at the veridical vibrotactile site. When the lights were presented distally from the vibrotactile site; meaning they were non colocated, the random group reverted to localising the tactile sensation at the initial, inaccurate location proximal to the veridical site. In contrast, when the structured group experienced the lights, distally non co-located at position 2 and 3 they retained the calibrated localisation at the veridical vibration site. There appeared to be a limit on this retention as when the light was located at position four on the fingers the visual capture response diminished and they started to localise the tactile sensation proximal once again from the veridical site. However, even as the visual capture diminished the distribution of response never reached the same extent as seen in their initial tactile localisation when a light was not present. It should be noted that the effect sizes for these results were small. In addition, the sample size for the demographic was also small, leading to potential issues with data diversity. As only eight people were used (four people in each group), caution needs to be taken when considering generalisability. Additional participants are required from a more diverse sample to externally validate.

Although careful measurements were taken to scale the virtual arms to match the participants own arms, there was an unexpected dissonance between where the vibrotactile actuators were physically located on their arm and where participants localised them on the virtual arm. This was evident in both the random and structured group when a visual stimulus did not accompany the vibration. They generally localised the vibrotactile sensation one section proximal from the veridical site. A possible reason for this observation may have been the appearance of the virtual limbs. Although the virtual arms were scaled to the correct size, there may have been perceptual inconsistencies with how the participants viewed and embodied them. The virtual arms lacked elbows, and this subsequently may have reduced the number of ways to infer where the vibration motors were. The only anatomical landmark provided to participants was the wrist. However, if a boundary for a proximal known anatomical landmark was provided such as an elbow, it is possible that tactile acuity may be increased. Another possible reason for proximal relocation may have been the false indications given to participants during the set up phase in an attempt to mask the true location of the vibration motors. However, false indications were only administered distal from the vibration sites meaning a proximal baseline is unlikely. Similar results have been reported demonstrating that tactile localisation can be perceived more proximal when a visual stimulus is occluded, suggesting there maybe psychophysical factors that need to be accounted for Badde et al. (2020). The inaccurate initial spatial localisation is not problematic to the overall results, as both groups are consistent, and the overall hypothesis is investigating whether a distal relocation is possible, which is a relative measurement.

Despite the discrepancies between the participants tactile mislocalisation and the veridical vibrotactile site; when a light was presented co-located with the vibrotactile actuator at position 1, tactile localisation to the veridical vibration site was significantly improved. This was evident in both the random and structured group. This relocation of tactile perception is 
likely attributed to a visual capture response as participants were displayed a bias toward the visual localisation over their initial tactile localisation. When the light was decoupled from the vibrotactile actuator in a spatially incongruent manner at position 2, 3, and 4, tactile localisation did not consistently follow the light. Instead, there was a significantly different response from the random group and the structured group. When the light was positioned at these distal locations, the random group regressed the tactile localisation to that experienced in the no-light condition. If visual capture only relied on visual dominance; where visual stimulus superseded tactile stimulation, it would have been expected that the tactile sensation moves to where the light was situated no matter the distance, when associated. However, this was not observed; Instead the random group generally switched back and forth between section 7 , when the light was spatially co-located and section 8 when the light was spatially non co located. This finding suggests visual dominance or a purely hierarchical organisation of sensory information is not the only component of sensory integration.

Conversely, the structured group retained their tactile localisation at the veridical vibrotactile site even when the lights were decoupled at more distal locations. Interestingly, when the light is present at position 4; instead of retaining the visual capture at the veridical vibrotactile site, localisation starts to diminish and regress proximally toward inaccurate initial tactile localisation when the light was not present. This suggests the retention of accurate tactile localisation at the vibrotactile site was not simply due to gradually establishing a better tactile localisation from cumulative exposure over time, but due to the visual stimulus retaining its effect over a greater distance until it reached a threshold distance. There are at least two possible explanations to this finding either a ventriloquist aftereffect was observed, or attention influenced the visual capture effects.

In the case of a ventriloquist aftereffect, there may have been a sufficient number of exposures at position 1; where the light was co-located at the veridical vibrotactile location, that an ongoing visual capture effect may have been observed even without the presence of the light for a finite duration (Frissen et al., 2012; Bosen et al., 2017). This finite duration may have been until the light reached light position 4 . This explanation would provide evidence that visual capture and in extension the ventriloquist aftereffect is not mutable due to data from position 4 showing a proximal trajectory. Further research is needed to verify whether duration was the largest factor in the retention of accurate tactile localisation. Another explanation for the increased retention exhibited in the structured group may be due to attentional differences between the random presentation and the structured presentation. Increased attention to specific stimuli has shown to influence the degree and intensity of visual capture response (Odegaard et al., 2016; Badde et al., 2020). The structured group would have been able to expect and predict the movement of the light stimulus better than the random group, thus influencing the way participants divided their attention between the tactile sensation and the visual stimulus. Further research is necessary to conclude if attention may play a more important role than exposure in the movement of a tactile sensation.

Although a distal ipsilateral distal relocation of a tactile sensation was not observed to the extent that may be necessary for phantom limb pain treatment, the results corroborate and extend findings from Samad and Shams (2018) and Niijima and Ogawa (2014), where they observed around a $40 \mathrm{~mm}$ displacement. Due to standardising the section numbers in this study, a comparison to related findings cannot be made, regarding the gross amount of displacement in millimetres. However, on average the sections of the grid were longer than $40 \mathrm{~mm}$ in the proximal/ distal plane. It is possible that the visual capture effects observed were larger than related studies. Further research is needed to quantify the distance relocated in a more granular fashion.

These results may be of use to other treatments such as graded motor imagery (GMI) in which tactile acuity is trained to improve. The structured groups results demonstrated that either a visual capture or ventriloquist aftereffect was established; however, even when the associated visual stimulus was moved, an accurate perception of where the vibrotactile sensation was located was retained. There is potential that the methods could be used to train tactile acuity without the concern that a visual capture response could potentially cause unintended results regarding tactile acuity.

\section{DATA AVAILABILITY STATEMENT}

The raw data supporting the conclusions of this article will be made available by the authors, without undue reservation. The open-source data is hosted on Figshare: https://doi.org/10.6084/ m9.figshare.14527068.v1 (Willis, 2021).

\section{ETHICS STATEMENT}

The studies involving human participants were reviewed and approved by University of Portsmouth - Faculty of the Creative and Cultural Industries Ethics Committee. The patients/ participants provided their written informed consent to participate in this study.

\section{AUTHOR CONTRIBUTIONS}

DW - First Authorship. BS - Senior Authorship, WP - Senior Authorship. DW, BS, and WP contributed to the idea of the project. DW and BS contributed to the methodology. DW created the virtual reality application and electronic peripherals, conducted the data collection, ran data analysis and wrote the majority of the paper. BS and WP contributed to the data analysis, structuring of the paper and reviewed and refined the paper. 


\section{REFERENCES}

Ackerley, R., Hassan, E., Curran, A., Wessberg, J., Olausson, H., and McGlone, F. (2012). An Fmri Study on Cortical Responses during Active Self-Touch and Passive Touch from Others. Front. Behav. Neurosci. 6, 51. doi:10.3389/fnbeh.2012.00051

Badde, S., Navarro, K. T., and Landy, M. S. (2020). Modality-specific Attention Attenuates Visual-Tactile Integration and Recalibration Effects by Reducing Prior Expectations of a Common Source for Vision and Touch. Cognition 197, 104170. doi:10.1016/j.cognition.2019.104170

Barghout, A., Cha, J., El Saddik, A., Kammerl, J., and Steinbach, E. (2009). Spatial Resolution of Vibrotactile Perception on the Human Forearm when Exploiting Funneling Illusion. 2009 IEEE International Workshop on Haptic Audio visual Environments and Games, Lecco, Italy, November 7-8, 2009. IEEE, 19-23.

Bosen, A. K., Fleming, J. T., Allen, P. D., O'Neill, W. E., and Paige, G. D. (2017). Accumulation and Decay of Visual Capture and the Ventriloquism Aftereffect Caused by Brief Audio-Visual Disparities. Exp. Brain Res. 235, 585-595. doi:10. 1007/s00221-016-4820-4

Carey, M., Crucianelli, L., Preston, C., and Fotopoulou, A. (2019). The Effect of Visual Capture towards Subjective Embodiment within the Full Body Illusion. Scientific Rep. 9, 1-12. doi:10.1038/s41598-019-39168-4

Dunn, J., Yeo, E., Moghaddampour, P., Chau, B., and Humbert, S. (2017). Virtual and Augmented Reality in the Treatment of Phantom Limb Pain: A Literature Review. Nre 40, 595-601. doi:10.3233/NRE-171447

Finn, S. B., Perry, B. N., Clasing, J. E., Walters, L. S., Jarzombek, S. L., Curran, S., et al. (2017). A Randomized, Controlled Trial of Mirror Therapy for Upper Extremity Phantom Limb Pain in Male Amputees. Front. Neurol. 8, 267. doi:10. 3389/fneur.2017.00267

Flor, H., and Diers, M. (2009). Sensorimotor Training and Cortical Reorganization. Nre 25, 19-27. doi:10.3233/NRE-2009-0496

Frissen, I., Vroomen, J., and de Gelder, B. (2012). The Aftereffects of Ventriloquism: the Time Course of the Visual Recalibration of Auditory Localization. Seeing and perceiving 25, 1-14. doi:10.1163/187847611x620883

Kuner, R., and Flor, H. (2017). Structural Plasticity and Reorganisation in Chronic Pain. Nat. Rev. Neurosci. 18, 20, 30. doi:10.1038/nrn.2016.162

Lamp, G., Goodin, P., Palmer, S., Low, E., Barutchu, A., and Carey, L. M. (2019). Activation of Bilateral Secondary Somatosensory Cortex with Right Hand Touch Stimulation: a Meta-Analysis of Functional Neuroimaging Studies. Front. Neurol. 9, 1129. doi:10.3389/fneur.2018.01129

Lee, H., Lee, J., Kim, C., Kim, G., Kim, E.-S., and Whang, M. (2015). Brain Process for Perception of the "Out of the Body" Tactile Illusion for Virtual Object Interaction. Sensors 15, 7913-7932. doi:10.3390/s150407913

Lee, M. W. L., McPhee, R. W., and Stringer, M. D. (2008). An Evidence-based Approach to Human Dermatomes. Clin. Anat. 21, 363-373. doi:10.1002/ca.20636

Moseley, L. G., Zalucki, N. M., and Wiech, K. (2008). Tactile Discrimination, but Not Tactile Stimulation Alone, Reduces Chronic Limb Pain. Pain 137, 600-608. doi:10.1016/j.pain.2007.10.021

Niijima, A., and Ogawa, T. (2014). A Study of Changing Locations of Vibrotactile Perception on a Forearm by Visual Stimulation. In International Conference on Collaboration Technologies (Springer), Santiago, Chile, September 8-10, 2014, 86-95. doi:10.1007/978-3-662-44651-5_8

Odegaard, B., Wozny, D. R., and Shams, L. (2016). The Effects of Selective and Divided Attention on Sensory Precision and Integration. Neurosci. Lett. 614, 24-28. doi:10.1016/j.neulet.2015.12.039

Ortiz-Catalan, M., Sander, N., Kristoffersen, M. B., Håkansson, B., and Brånemark, R. (2014). Treatment of Phantom Limb Pain (Plp) Based on Augmented Reality and Gaming Controlled by Myoelectric Pattern Recognition: a Case Study of a Chronic Plp Patient. Front. Neurosci. 8. doi:10.3389/fnins.2014.00024

Osumi, M., Inomata, K., Inoue, Y., Otake, Y., Morioka, S., and Sumitani, M. (2019). Characteristics of Phantom Limb Pain Alleviated with Virtual Reality Rehabilitation. Pain Med. 20, 1038-1046. doi:10.1093/pm/pny269
Osumi, M., Sano, Y., Ichinose, A., Wake, N., Yozu, A., Kumagaya, S.-I., et al. (2020). Direct Evidence of Eeg Coherence in Alleviating Phantom Limb Pain by Virtual Referred Sensation: Case Report. Neurocase 26, 55-59. doi:10.1080/13554794. 2019.1696368

Perry, B. N., Armiger, R. S., Wolde, M., McFarland, K. A., Alphonso, A. L., Monson, B. T., et al. (2018). Clinical Trial of the Virtual Integration Environment to Treat Phantom Limb Pain with Upper Extremity Amputation. Front. Neurol. 9, 770. doi:10.3389/fneur.2018.00770

Pirowska, A., Wloch, T., Nowobilski, R., Plaszewski, M., Hocini, A., and Ménager, D. (2014). Phantom Phenomena and Body Scheme after Limb Amputation: A Literature Review. Neurologia i neurochirurgia polska 48, 52-59. doi:10.1016/j. pjnns.2013.03.002

Purves, D. (2018). Neuroscience. 6th edition edn. New York: Oxford University Press.@2018.

Richardson, C., and Kulkarni, J. (2017). A Review of the Management of Phantom Limb Pain: Challenges and Solutions. Jpr Vol. 10, 1861-1870. doi:10.2147/JPR.S124664

Samad, M., and Shams, L. (2018). Recalibrating the Body: Visuotactile Ventriloquism Aftereffect. PeerJ 6, e4504. doi:10.7717/peerj.4504

Sano, Y., Wake, N., Ichinose, A., Osumi, M., Oya, R., Sumitani, M., et al. (2016). Tactile Feedback for Relief of Deafferentation Pain Using Virtual Reality System: a Pilot Study. J. Neuroengineering Rehabil. 13, 61. doi:10.1186/ s12984-016-0161-6

Shankar, H., Hansen, J., and Thomas, K. (2015). Phantom Pain in a Patient with Brachial Plexus Avulsion Injury: Table 1. Pain Med. 16, 777-781. doi:10.1111/ pme. 12635

Simões-Franklin, C., Whitaker, T. A., and Newell, F. N. (2011). Active and Passive Touch Differentially Activate Somatosensory Cortex in Texture Perception. Hum. Brain Mapp. 32, 1067-1080. doi:10.1002/hbm.21091

Ueda, S., Uchida, M., Nozawa, A., and Ide, H. (2008). A Tactile Display Using Phantom Sensation with Apparent Movement Together. Electron. Comm. Jpn. 91, 29-38. doi:10.1002/ecj.10000

Wake, N., Sano, Y., Oya, R., Sumitani, M., Kumagaya, S.-i., and Kuniyoshi, Y. (2015). Multimodal Virtual Reality Platform for the Rehabilitation of Phantom Limb Pain, Neural Engineering (NER), 2015 7th International IEEE/EMBS Conference on, Montpellier, France, April 22-24, 2015. IEEE, 787-790. doi:10. 1109/NER.2015.7146741

Weeks, S. R., Anderson-Barnes, V. C., and Tsao, J. W. (2010). Phantom Limb Pain. The neurologist 16, 277-286. doi:10.1097/NRL.0b013e3181edf128

Willis, D., Powell, W., Powell, V., and Stevens, B. (2019). Visual Stimulus Disrupts the Spatial Localization of a Tactile Sensation in Virtual Reality. In 2019 IEEE Conference on Virtual Reality and 3D User Interfaces (VR), Osaka, Japan, March 23-27, 2019. 484-491.

Willis, D., Stevens, B., and Powell, W. (2021). Visual capture of a tactile sensation is influenced by repeated, structured exposure of a visual stimulus in virtual reality - Random_Cycle_Sequenced SPSS Source Data. Dataset. doi:10.6084/ m9.figshare.14527068.v1

Zhang, J. J. Q., Fong, K. N. K., Welage, N., and Liu, K. P. Y. (2018). The Activation of the Mirror Neuron System during Action Observation and Action Execution with Mirror Visual Feedback in Stroke: A Systematic Review, Neural Plasticity, 2018, 1, 14. doi:10.1155/2018/2321045

Conflict of Interest: The authors declare that the research was conducted in the absence of any commercial or financial relationships that could be construed as a potential conflict of interest.

Copyright (c) 2021 Willis, Stevens and Powell. This is an open-access article distributed under the terms of the Creative Commons Attribution License (CC $B Y)$. The use, distribution or reproduction in other forums is permitted, provided the original author(s) and the copyright owner(s) are credited and that the original publication in this journal is cited, in accordance with accepted academic practice. No use, distribution or reproduction is permitted which does not comply with these terms. 